\title{
Cutaneous sarcoidosis of the scalp unmasking systemic involvement: A case report
}

\author{
DANIEL BODA $^{1,2}$, ANA CUTOIU ${ }^{3}$, NONA BEJENARIU ${ }^{4}$ and CONSTANTIN CARUNTU ${ }^{2}$ \\ ${ }^{1}$ Department of Dermatology, 'Ponderas' Academic Hospital, 014142 Bucharest; ${ }^{2}$ Department of Dermatology, \\ 'Carol Davila' University of Medicine and Pharmacy, 050474 Bucharest; \\ ${ }^{3}$ Department of Dermatology, 'Colentina' Clinical Hospital, 020125 Bucharest; ${ }^{4}$ Department of \\ Pathology, 'Santomar' Laboratory, 400350 Cluj Napoca, Romania
}

Received July 23, 2021; Accepted August 23, 2021

DOI: $10.3892 /$ etm.2021.10803

\begin{abstract}
Sarcoidosis is a multisystemic granulomatous disease of unknown cause that affects any organ, especially the lungs, eyes, lymph nodes and skin. Skin sarcoidosis occurs in about one-fourth of patients with systemic disease and may also arise in isolation. Skin lesions are divided into two groups, as follows: specific skin lesions where histologic examination shows the typical sarcoid granulomas and non-specific skin lesions. Specific lesions are lupus pernio, infiltrated plaques, maculopapular eruptions, subcutaneous nodules and scars. The most significant non-specific skin lesion seen in sarcoidosis is erythema nodosum. Cutaneous sarcoidosis is known as the 'great imitator' in dermatology, because it can mimic a vast variety of cutaneous lesions. The diagnosis of sarcoidosis is made by exclusion and is supported by the recognition of specific clinical features, the detection of classic histopathologic findings and the exclusion of other granulomatous diseases. We present a case report concerning a single, solitary and asymptomatic lesion on the scalp.
\end{abstract}

\section{Introduction}

Sarcoidosis represents a rare condition, affecting several organs. Its etiology, defined by the development of non-caseating granulomas in the affected organs, is still unknown (1). The organ most commonly affected by sarcoidosis is the lung; however, there is also skin involvement in $20-35 \%$ of the patients with systemic conditions and it can be the initial presentation as well. This disease affects both sexes of all ages and races; nonetheless, it occurs more commonly and severely in women and black individuals. Thus far, it is still

Correspondence to: Dr Ana Cutoiu, Department of Dermatology, 'Colentina' Clinical Hospital, 19-21 Stefan cel Mare Street, 020125 Bucharest, Romania

E-mail: ana.cutoiu@yahoo.com

Key words: sarcoidosis, granulomatous disease, dermoscopy, reflectance confocal microscopy, cutaneous, skin lesion unclear what the cause of sarcoidosis is, even though possible infectious, immunological, genetic and environmental factors are to be considered $(2,3)$. The skin lesions are either specific skin lesions, where histologic examination indicates the characteristic sarcoid granulomas, or non-specific skin lesions. Maculopapular eruptions, subcutaneous nodules, lupus pernio, scars and infiltrated plaques constitute specific lesions. The main non-specific skin lesion of sarcoidosis is erythema nodosum. Patients with a chronic course of the disease (scar infiltration, lupus pernio, skin plaques) present parenchymal involvement more frequently than those with acute sarcoidosis, which a better prognosis (maculopapular rash, erythema nodosum) (1).

\section{Case report}

A 35-year-old man presented at the Department of Dermatology of Ponderas Academic Hospital, Bucharest, Romania for an orange-red indurated plaque with a raised, shiny border on the scalp, which appeared suddenly during the last 6 months (Fig. 1). The patient did not report any significant changes, bleeding or itch. However, for the previous 10 days, he had developed a dry cough, along with malaise, night sweats and shortness of breath on medium exertion. The patient denied smoking and exposure to any toxic chemicals. Polarized dermoscopy (using DermLite, DL4, x10) with immersion oil showed diffuse monomorphic linear vessels, as well as yellow-orange and pink structureless areas (Fig. 2). The diascopy revealed the characteristic 'apple jelly' nodules (Fig. 3). The reflectance confocal microscopy of the lesion showed superficial tortuous vessels, many reticulin fibers and inflammatory cells (Fig. 4). A biopsy was performed. Subsequent histopathology revealed chronic dermal inflammation with multiple confluent non-caseating granulomas with epithelioid cells, lymphocytes and multinucleated giant cells (Fig. 5C). The blood tests showed no abnormalities, except for a high level of angiotensin converting enzyme (141 U/1) and a slightly elevated C-reactive protein $(0.75 \mathrm{mg} / \mathrm{dl})$. In addition, the tests for Mycobacterium tuberculosis were negative.

After complete excision of the lesion with narrow margins, the patient was referred to the Pneumology Department for 


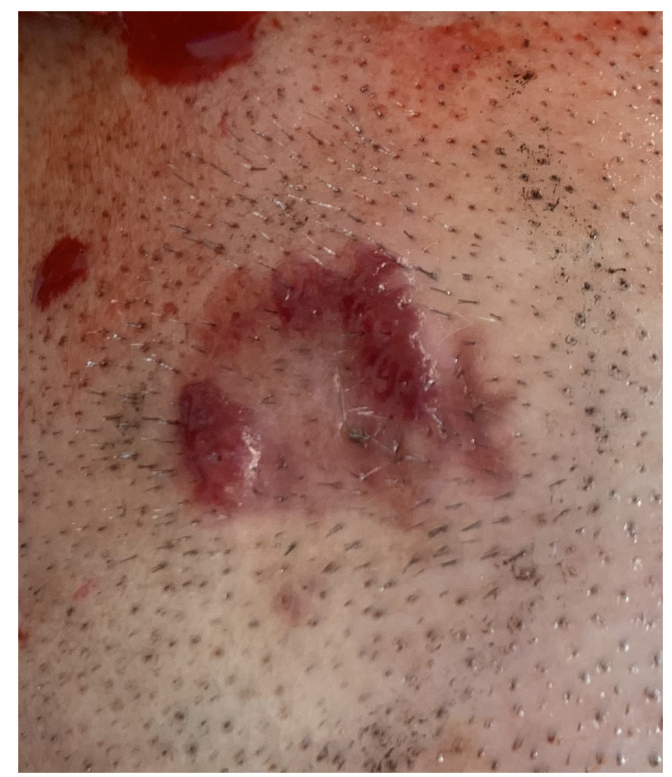

Figure 1. Clinical image of cutaneous sarcoidosis after biopsy. Infiltrated red-orange plaque on the scalp.

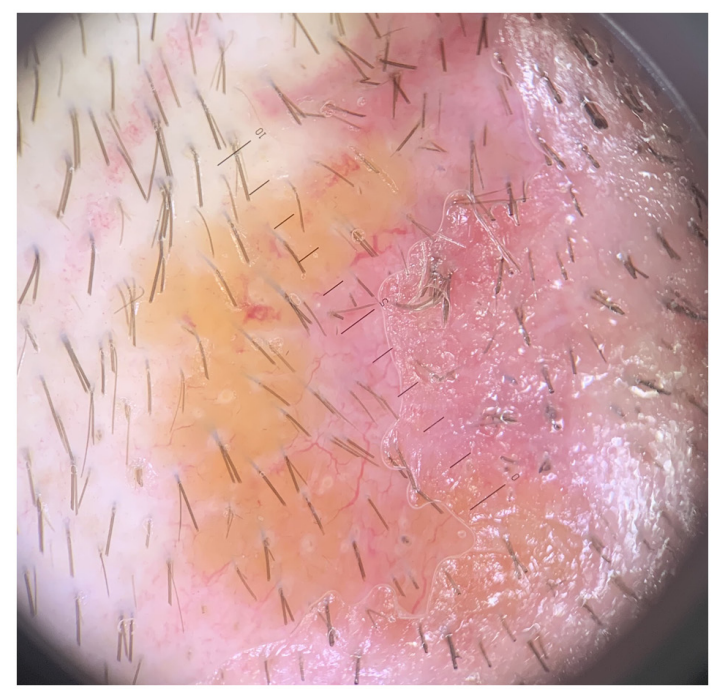

Figure 2. Dermoscopy of cutaneous sarcoidosis. Linear vessels over a yellow-orange translucent structureless background.

further examination and a CT scan of the thorax, abdomen and pelvis with contrast. It revealed multiple bilateral mediastinal lymphadenopathy $(23 / 24 \mathrm{~mm}$, right superior paratracheal lymph node; 34/32 $\mathrm{mm}$, right inferior paratracheal lymph node; $33 / 24 \mathrm{~mm}$, lateral aortic lymph node; $28 / 26 \mathrm{~mm}$, right hilar lymph node; $28 / 24 \mathrm{~mm}$, left hilar lymph node) and multiple symmetric pulmonary micronodules with peribronchovascular distribution (Fig. 6A and B). In addition, the patient was diagnosed with secondary increased airway hyperreactivity. The ENT examination showed no pathologic changes. The patient was then referred to the cardiologist and the ophthalmologist for a thorough evaluation.

The patient was diagnosed with systemic sarcoidosis and started systemic treatment with methylprednisolone $32 \mathrm{mg}$ and inhalation therapy with beclomethasone/formoterol $100 / 6 \mathrm{mg}$.

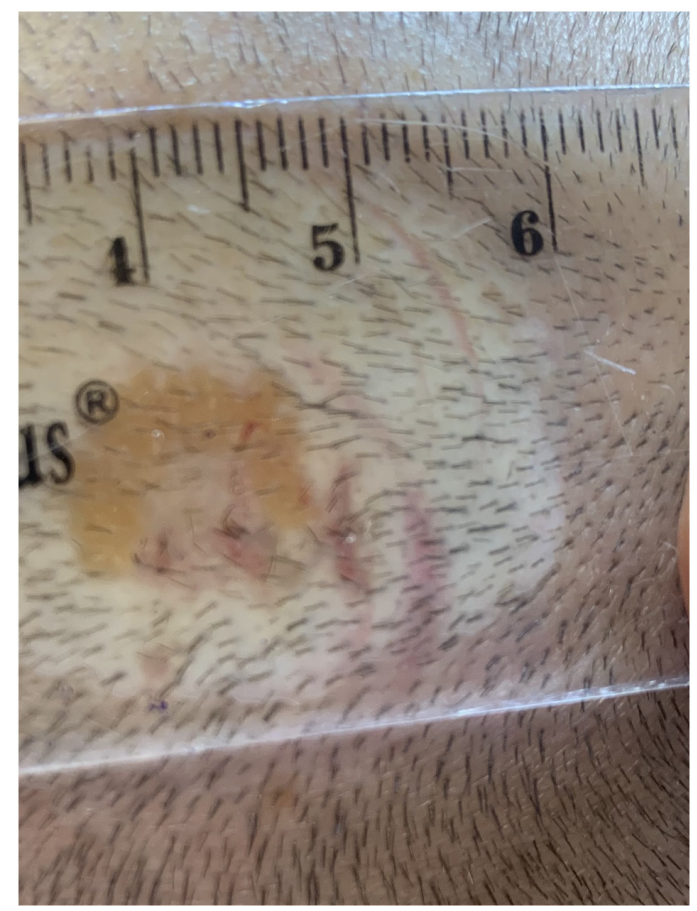

Figure 3. Diascopy of cutaneous sarcoidosis. ‘Apple jelly’ nodules.

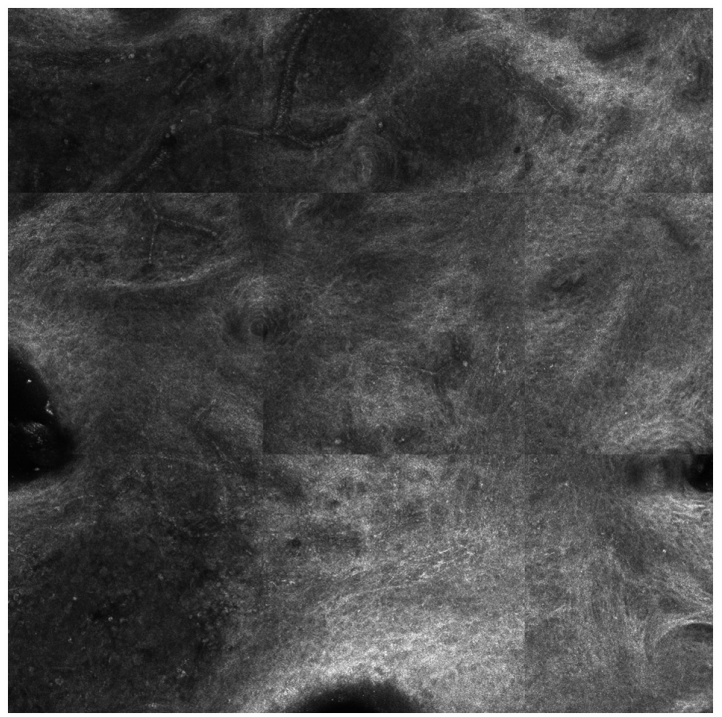

Figure 4. Reflectance confocal microscopy of cutaneous sarcoidosis. Superficial tortuous vessels, many reticulin fibers and inflammatory cells.

\section{Discussion}

Sarcoidosis represents a chronic granulomatous condition, which is characterized by non-necrotizing granulomas. It may affect any organ and sometimes constitutes a considerable diagnostic challenge. The organ most commonly affected by sarcoidosis is the lung; however, there is also skin involvement in 20-35\% of the patients with systemic conditions and it can be the initial presentation as well. Cutaneous sarcoidosis affects mostly the face and the limbs (2). Cutaneous sarcoidosis is also known as the 'great imitator' in dermatology, since it can mimic a great variety of cutaneous lesions (4). 

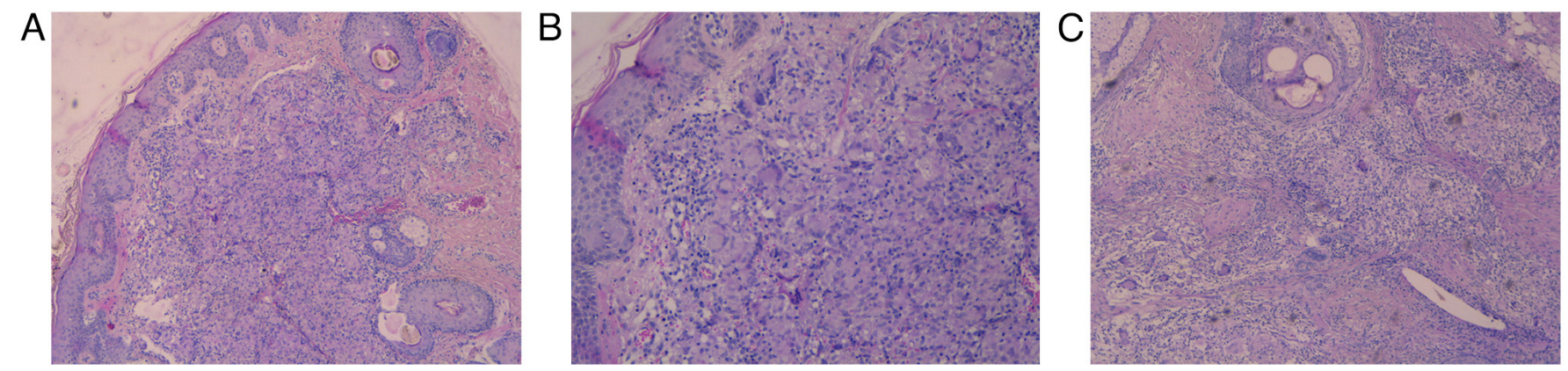

Figure 5. (A-C) Histopathology of cutaneous sarcoidosis. Chronic dermal inflammation with multiple confluent non-caseating granulomas with epithelioid cells, lymphocytes and multinucleated giant cells.
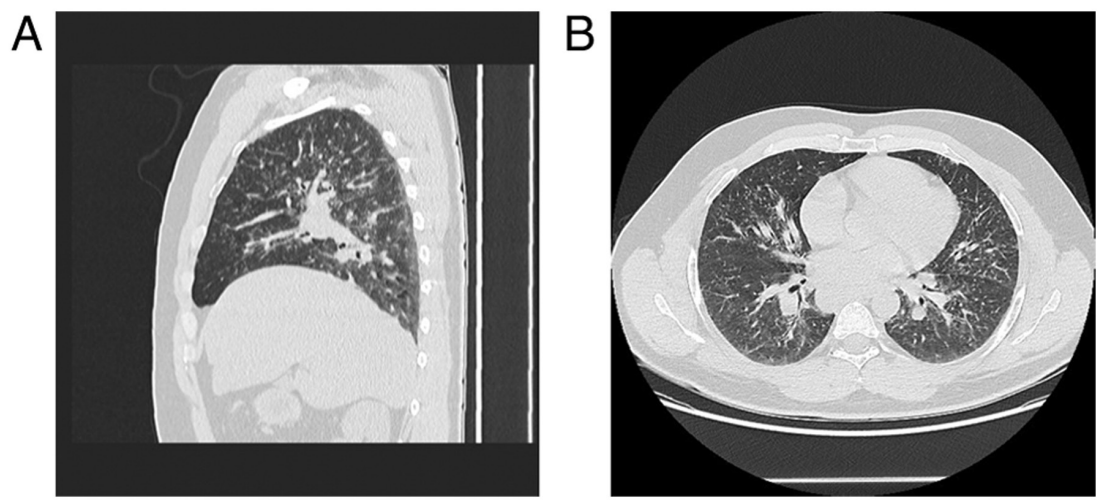

Figure 6. (A and B) Computed tomography (CT) scans of pulmonary sarcoidosis. Multiple bilateral mediastinal lymphadenopathy and symmetric pulmonary micronodules with peribronchovascular distribution.

The skin lesions are either specific skin lesions where histologic examination indicates the characteristic sarcoid granulomas, or non-specific skin lesions. Maculopapular eruptions, subcutaneous nodules, lupus pernio, scars and infiltrated plaques constitute specific lesions. The main non-specific skin lesion of sarcoidosis is erythema nodosum, which can be typically seen in young women as a marker of acute sarcoidosis $(1,2)$. Conversely, lupus pernio is generally associated with chronic sarcoidosis, affecting women and older patients. Scar sarcoidosis may occur on tattoos, surgical scars or vaccination sites. Plaque sarcoidosis typically involves the limbs, being an indolent form of the condition (2). Parenchymal involvement occurs more in patients with a chronic course of the disease (lupus pernio, scar infiltration and skin plaques) as opposed to those with acute sarcoidosis (1).

Sarcoidosis can be diagnosed by exclusion and is backed by recognizing the specific clinical characteristics, detecting the typical histopathologic findings and excluding other granulomatous conditions (fungal infection, tuberculosis, leishmaniasis, foreign body reactions and rheumatoid nodules) $(2,5)$. From a clinical point of view, diascopy can prove helpful in displaying the granulomatous inflammation. This technique is performed by pressing a glass slide against the skin, which allows the potential 'apple jelly' nodules to be seen (2).

Compulsory standard investigations, accompanied by physical examination and a complete medical history, should include pulmonary function tests, chest X-ray, ophthalmologic evaluation, electrocardiogram, biochemistry, full blood count, serum immunoglobulins, as well as a $24-\mathrm{h}$ urinary calcium assay (4). The measurement of the serum angiotensin-converting enzyme (ACE), produced by sarcoidal granulomas, can prove helpful in monitoring the development of the disease. However, it is not a very useful diagnostic test, considering that the levels may be raised in other diseases, such as alcoholic liver disease and diabetes (2). Specific cutaneous lesions, together with elevated serum ACE levels, high $\mathrm{CD}_{4} / \mathrm{CD}_{8}$ ratio and bronchoalveolar lavage lymphocytosis may act as predictors of progressive disease in sarcoidosis (6). When there is lung involvement, physicians should apply the classification of chest X-rays by De Remee: stage I, bilateral hilar lymphadenopathy (BHL); stage II, BHL plus pulmonary parenchymal infiltration; stage III, parenchymal involvement infiltration without BHL (7). In addition, patients with parenchymal involvement may experience a restrictive pattern of lung impairment, as well as increased airway hyperreactivity (8).

Dermoscopy represents a non-invasive tool that enables the visualization of vascular and pigmented structures not visible to the naked eye. Dermoscopy is conventionally used for skin tumor diagnosis. However, it has gained an increased interest during the past years as a useful tool in general dermatology in the clinical diagnosis of inflammatory and infectious skin manifestations. Dermoscopy is valuable in differentiating an extensive range of granulomatous inflammatory and infectious skin conditions, such as necrobiosis lipoidica, granuloma annulare, cutaneous leishmaniasis, lupus vulgaris, syphilis, foreign body reactions, atypical mycobacteriosis, fungal 
infections or rheumatoid nodules, as well as skin tumors, such as sebaceous adenoma or trichoepithelioma, as sarcoidosis can clinically mimic all of these lesions (9). Nevertheless, dermoscopy does not seem to be sufficient in order to accurately establish the diagnosis of cutaneous sarcoidosis, since a suspicious lesion of cutaneous sarcoidosis displays linear vessels and yellow-orange structureless areas, but so does any granulomatous condition $(9,10)$. Hence, conventional methods such as radiography, laboratory tests or histopathology represent the state of the art in the diagnosis of sarcoidosis (9).

Reflectance confocal microscopy has been recently used as support for the diagnosis of cutaneous sarcoidosis. It represents a non-invasive imaging technique that allows in vivo visualization of the papillary dermis and epidermis with cellular level resolution. Granulomatous conditions, such as sarcoidosis, could be evaluated using this technique. Identifying bright beaded-like structures that correspond to reticulin fibers overlying granulomas can prove very helpful, in association with dermoscopy, in establishing the diagnosis of sarcoidosis (11).

Considering the ease with which to perform a biopsy for skin lesions, it generally represents the conventional type of investigation. The biopsy specimens usually show a dermal infiltrate of non-caseating granulomas, made of epithelioid cells, multinucleate giant cells and a thin peripheral rim of lymphocytes. Deep fungal and mycobacterial infections should be excluded by special stains and culture (2).

Treating cutaneous sarcoidosis can often become frustrating, since the lesions can either be refractory to treatment or recur after successful treatment. Intralesional or topical steroids are used for localized skin involvement. Intralesional steroids (triamcinolone acetonide $5 \mathrm{mg} / \mathrm{ml}$, with injections repeated at 2-3 week intervals) are usually more effective, because even very potent topical steroids do not penetrate the skin lesion effectively $(5,8)$.

For progressive and multiple lesions and/or systemic symptoms, systemic treatments are used. Systemic glucocorticoids are reported to be the most effective agent (used at slow, tapering dosages, starting at 20-40 mg of oral prednisone daily for four to six weeks); however, there are many patients that do not respond well to steroids. In refractory patients, methotrexate, hydroxychloroquine and thalidomide can prove effective (4). Tofacitinib, adalimumab, etanercept, pentoxifylline, apremilast, infliximab, and even topical photodynamic therapy represent several new therapeutic options $(8,12-15)$. Nonetheless, the globally acknowledged standard therapies include the administration of corticosteroids, methotrexate and antimalarials, considering the need to perform more studies for the above-mentioned treatments (8).

Most types of cutaneous sarcoidosis present a chronic course, except maculopapular sarcoidosis and erythema nodosum. The general prognosis of the disease is linked to the severity and the extent of the internal involvement (2).

Considering the fact that cutaneous sarcoidosis is often a precursor of the systemic form, it is of paramount importance to diagnose correctly as well as any form of cutaneous sarcoidosis as early as possible. Reflectance confocal microscopy and dermoscopy represent very useful tools; however, histologic examination remains the investigation of choice. In addition, interdisciplinary collaboration between various medical specialties is required in these cases. In our case, the patient's test results showed similar aspects with the ones depicted in the literature.

\section{Acknowledgements}

Not applicable.

\section{Funding}

No funding was received.

\section{Availability of data and materials}

All data and materials supporting the results of the present study are available in the published article.

\section{Authors' contributions}

DB performed the biopsy and the excision of the lesion and participated in the therapeutic management of the case study. AC performed the diascopy and the dermoscopy examination and performed critical review of the literature findings. $\mathrm{CC}$ performed the confocal microscopy examination and performed critical review of the literature findings. NB performed the histopathologic examination. All authors read and approved the final manuscript for publication.

\section{Ethics approval and consent to participate}

There is a general valid ethics approval for this case presentation that is part of the PATHDERM project no 61PCCDI/2018 (PN-III-P1-1.2-PCCDI-2017-0341).

\section{Patient consent for publication}

A signed consent for clinical examination, surgery, other medical investigations, treatment and capturing images for publication was obtained from the patient.

\section{Competing interests}

The authors declare that they have no competing interests.

\section{References}

1. Yanardağ H, Pamuk ON and Karayel T: Cutaneous involvement in sarcoidosis: Analysis of the features in 170 patients. Respir Med 97: 978-982, 2003

2. Wilson NJ and King CM: Cutaneous sarcoidosis. Postgrad Med J 74: 649-652, 1998.

3. Ungprasert P, Wetter DA, Crowson CS and Matteson EL: Epidemiology of cutaneous sarcoidosis, 1976-2013: A population-based study from Olmsted County, Minnesota. J Eur Acad Dermatol Venereol 30: 1799-1804, 2016.

4. Katta R: Cutaneous sarcoidosis: A dermatologic masquerader. Am Fam Physician 65: 1581-1584, 2002.

5. Vasaghi A and Kalafi A: Unusual Manifestation of cutaneous sarcoidosis: A case report of morpheaform sarcoidosis. Acta Med Iran 50: 648-651, 2012.

6. Yanardag H, Tetikkurt C, Bilir M, Demirci S and Iscimen A: Diagnosis of cutaneous sarcoidosis; clinical and the prognostic significance of skin lesions. Multidiscip Respir Med 8: 26, 2013.

7. De Remee RA: The roentgenographic staging of sarcoidosis. Historic and contemporary perspectives. Chest 1: 128-133, 1983. 
8. Choi SC, Kim HJ, Kim CR, Byun JI, Lee DY, Lee JH, Lee ES and Yang JM: A case of morpheaform sarcoidosis. Ann Dermatol 22: 316-318, 2010.

9. Pellicano R, Tiodorovic-Zivkovic R, Gourhant JY, Catricalà C, Ferrara G, Caldarola G, Argenziano G and Zalaudek I: Dermoscopy of cutaneous sarcoidosis. Dermatology 221: 51-54, 2010.

10. Conforti C, Giuffrida R, de Barros MH, Resende FSS, Cerroni L and Zalaudek I: Dermoscopy of a single plaque on the face: An uncommon presentation of cutaneous sarcoidosis. Dermatol Pract Concept 8: 174-176, 2018.

11. Pasquali P, Gonzalez S, Fortuño A and Freites-Martinez A: In-vivo assessment of a case of cutaneous sarcoidosis using reflectance confocal microscopy. An Bras Dermatol 94: 93-95, 2019.

12. Heffernan MP and Smith DI: Adalimumab for treatment of cutaneous sarcoidosis. Arch Dermatol 142: 17-19, 2006.
13. Baughman RP, Judson MA, Ingledue R, Craft NL and Lower EE: Efficacy and safety of apremilast in chronic cutaneous sarcoidosis. Arch Dermatol 148: 262-264, 2012.

14. Damsky W, Thakral D, Emeagwali N, Galan A and King B Tofacitinib treatment and molecular analysis of cutaneous sarcoidosis. N Engl J Med 379: 2540-2546, 2018.

15. Karrer S, Abels C, Wimmershoff MB, Landthaler M and Szeimies RM: Successful treatment of cutaneous sarcoidosis using topical photodynamic therapy. Arch Dermatol 138: 581-584, 2002

(7) $\odot$ This work is licensed under a Creative Commons Attribution-NonCommercial-NoDerivatives 4.0 International (CC BY-NC-ND 4.0) License. 